\title{
Pion form factors in the $\varepsilon$ regime
}

\author{
JLQCD Collaboration: H. Fukaya ${ }^{* a \dagger}$ S. Aoki ${ }^{b, c}$, S. Hashimoto ${ }^{d, e}$, T. Kaneko ${ }^{d, e}$, \\ H. Matsufuru ${ }^{d, e}$, J. Noaki ${ }^{d}$, T. Onogi ${ }^{a}$ and N. Yamada ${ }^{d, e}$ \\ ${ }^{a}$ Department of Physics, Osaka University, Toyonaka, Osaka 560-0043 Japan \\ ${ }^{b}$ Graduate School of Pure and Applied Sciences, University of Tsukuba, Tsukuba 305-8571, \\ Japan \\ ${ }^{c}$ Center for Computational Sciences, University of Tsukuba, Tsukuba 305-8577, Japan \\ ${ }^{d}$ High Energy Accelerator Research Organization (KEK), Tsukuba 305-0801, Japan \\ e School of High Energy Accelerator Science, The Graduate University for Advanced Studies \\ (Sokendai), Tsukuba 305-0801, Japan
}

We consider how to extract the pion form factors in the $\varepsilon$ regime. Using the correlators with nonzero momenta and taking appropriate ratios of them, we eliminate the dominant finite volume effect from the zero-momentum pion mode. Our preliminary lattice result for the pion charge radius is consistent with the experiment.

The 30 International Symposium on Lattice Field Theory - Lattice 2012,

June 24-29, 2012

Cairns, Australia

\footnotetext{
* Speaker.

†E-mail: hfukaya@het.phys.sci.osaka-u.ac.jp
} 


\section{Introduction}

JLQCD collaboration has been performing lattice QCD simulations with dynamical overlap fermions [四]. The exact chiral symmetry realized on the lattice by the overlap fermions, not only enables us to analyze QCD in a theoretically clean way, but also makes the hybrid Monte Carlo updates fairly stable even in the vicinity of the chiral limit. In fact, we have succeeded in simulating QCD with the pion mass below its physical point [2, 目, 田, 回].

A drawback of the use of the overlap fermion action is its high numerical cost. We therefore had to choose a rather small lattice size $L \sim 1.8 \mathrm{fm}$, except for two main simulations with the pion masses around $290 \mathrm{MeV}$ and $380 \mathrm{MeV}$, for which we set $L \sim 2.7 \mathrm{fm}$. In particular, our simulation with the lightest pion mass $\sim 100 \mathrm{MeV}$ is inside the so-called $\varepsilon$ regime [ 6$]$, where the correlation length of the pion exceeds the lattice size $L$.

In the $\varepsilon$ regime, the finite volume effects become large, so that the physics is largely distorted from the infinite volume limit. Such finite volume effects are mainly due to the pion's zero-momentum mode, and can be described well within chiral perturbation theory (ChPT) with the zero-mode treated non-perturbatively. We have found that the chiral perturbation formulas for the Dirac eigenmode density [ $[$ ], and that for the pseudoscalar two point functions [ [ $]$ ], nicely describe our lattice data. We have extracted the chiral condensate [䧃], as well as the pion mass and decay constant [可].

A similar analysis using the chiral expansion is possible also for the three(or more)-point functions [9]. It is, however, expected that the contribution from the pion zero-mode become more complicated containing non-trivial combinations of (modified) Bessel functions, as is already seen in the one- and two-point functions.

In this work on the pion form factors, we propose a different direction, or a greatly simplified way of analysis in the $\varepsilon$ regime of QCD. The key ideas are

1. To insert non-zero momenta to relevant operators.

2. To take appropriate ratios of them.

Then, we can automatically eliminate the leading $\mathscr{O}(1)$ finite volume effects. Namely, we can extract the pion form factors without relying on non-trivial Bessel functions, as in the perturbative regime ( $p$ regime) [ए]]. Of course, this cancellation happens only at the leading order, and the nextto-leading order terms may contain non-trivial zero-mode contributions. But we will show here that the tree-level calculation already describe our lattice data for the vector form factor reasonably well, yielding a consistent value of the pion charge radius with the experiment.

\section{Two-point functions in the $\varepsilon$ regime}

Before considering the three-point functions, let us begin with a simpler case with the twopoint correlators to illustrate our new idea. For simplicity, we consider a two-flavor theory in a finite volume $V=L^{3} T$, with a degenerate quark mass $m$. The boundary condition is set periodic in every direction. We denote the chiral condensate by $\Sigma$ and the pion decay constant by $F$. Note that including the (sea) strange quark is not difficult [W] and does not change the following results at the leading order of ChPT. 
The $\varepsilon$ expansion of ChPT is expressed by an exact group integration of the pion's zeromomentum mode, as well as perturbative non-zero mode fields, where their mass is treated as a perturbation. Namely, the theory is a hybrid system of an $S U(2)$ matrix model (or a U(2) matrix model when the global topological charge of the gauge fields is fixed.), and massless fields.

The pseudoscalar correlator separated by a four vector $x=\left(t, x_{1}, x_{2}, x_{3}\right)$ is, thus, given by [ए]]

$$
\langle P(x) P(0)\rangle=X+Y\left(\frac{1}{V} \sum_{p \neq 0} \frac{e^{i p x}}{p^{2}}\right)+Z\left(\frac{1}{V} \sum_{p \neq 0} \frac{e^{i p x}}{\left(p^{2}\right)^{2}}\right)+\cdots,
$$

where $X, Y, Z \cdots$ denote (dimensionful) constants, including non-trivial (Bessel) functions of $m \Sigma V$ arising from the pion zero-mode. The propagator-like forms are contributions from the non-zero modes. Note that the only non-zero momenta are summed.

From the massless boson-like structure, it is not surprising to see that the zero-momentum correlator is a polynomial function of $t$. This is quite different from a conventional hyperbolic cosine function in the $p$ regime. In fact, this special feature of the $\varepsilon$ expansion, the existence of the constant term in particular, has been used for extracting the low-energy constants from finite

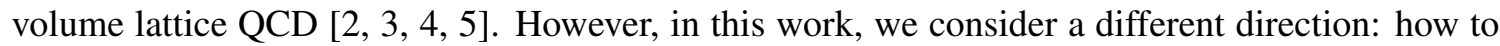
reduce such peculiarity in the $\varepsilon$ regime.

The answer is given by two steps. The first step is to insert a non-zero spatial momentum $\mathbf{p}$ :

$$
C_{P P}^{2 \mathrm{pt}}(t, \mathbf{p}) \equiv \int d^{3} x e^{-i \mathbf{p} \cdot \mathbf{x}}\langle P(x) P(0)\rangle=Y \frac{1}{2 E(\mathbf{p}) \sinh (E(\mathbf{p}) T / 2)} \cosh (E(\mathbf{p})(t-T / 2))+\cdots,
$$

where $E(\mathbf{p})=|\mathbf{p}|$. Note that the constant $X$ automatically vanishes, and the leading term has the same hyperbolic cosine form as in the $p$ expansion, except for an over-all coefficient $Y$. The second step is to take the ratio of them with different momenta: $C_{P P}^{2 \mathrm{pt}}(t, \mathbf{p}) / C_{P P}^{2 \mathrm{pt}}\left(t, \mathbf{p}^{\prime}\right)$. Then we can eliminate $Y$ which contains Bessel functions.

Note that the sub-leading terms, expressed by ellipses, still contain the contribution from the zero-momentum mode. But it is suppressed typically by $\sim 1 / 4 \pi F^{2} \sqrt{V}$.

In this way, contribution from the zero-momentum pion mode can be eliminated. It is reasonable that having a non-zero momentum reduces the information of the zero-mode.

\section{Three-point functions in the $\varepsilon$ regime}

Next, we consider three point operators all put at different points : $(x \neq y \neq z)$. In the $\varepsilon$ expansion, the pseudoscalar-vector(in 0-direction)-pseudoscalar function is given by

$$
\begin{aligned}
\left\langle P(x) V_{0}(y) P(z)\right\rangle= & A \frac{1}{V} \sum_{p \neq 0} \frac{i p_{0}}{p^{2}}\left(e^{i p(x-y)}+e^{i p(y-z)}\right) \\
& +B \frac{1}{V} \sum_{p \neq 0} \frac{1}{V} \sum_{p^{\prime} \neq 0} \frac{\left(i p_{0}+i p_{0}^{\prime}\right) e^{i p(x-y)} e^{i p^{\prime}(y-z)}}{p^{2} p^{\prime 2}} F_{V}\left(\left(p-p^{\prime}\right)^{2}\right)+\cdots,
\end{aligned}
$$

where $F_{V}$ denotes the vector form factor, and $A, B, \cdots$ denote (dimensionful) constants, including the non-trivial contributions from the pion zero-mode. Note in this case, that the constant term does not exist because a "vector" cannot be made from the zero-momentum mode alone.

In the following, we consider inserting the initial (spatial) momentum $\mathbf{p}_{i}$ to $P(x)$, and final momentum $\mathbf{p}_{f}$ to $P(z)$, splitting the discussion into three different cases. 
Case 1: $\mathbf{p}_{i} \neq 0, \mathbf{p}_{f} \neq 0$

In this case, it is straightforward to obtain

$$
\begin{aligned}
& C_{P V P}^{3 \mathrm{pt}}\left(t, t^{\prime} ; \mathbf{p}_{i}, \mathbf{p}_{f}\right) \equiv \int d^{3} x e^{-i \mathbf{p}_{i} \cdot \mathbf{x}} \int d^{3} z e^{i \mathbf{p}_{f} \cdot \mathbf{z}}\left\langle P(x) V_{0}(y) P(z)\right\rangle=B F_{V}\left(q^{2}\right) \\
& \quad \times\left[E\left(\mathbf{p}_{i}\right)+E\left(\mathbf{p}_{f}\right)\right]\left(\frac{\cosh \left(E\left(\mathbf{p}_{i}\right)(t-T / 2)\right)}{2 E\left(\mathbf{p}_{i}\right) \sinh \left(E\left(\mathbf{p}_{i}\right) T / 2\right)}\right)\left(\frac{\cosh \left(E\left(\mathbf{p}_{f}\right)\left(t^{\prime}-T / 2\right)\right)}{2 E\left(\mathbf{p}_{f}\right) \sinh \left(E\left(\mathbf{p}_{f}\right) T / 2\right)}\right)+\cdots,
\end{aligned}
$$

where $q^{2}=\left(\mathbf{p}_{i}-\mathbf{p}_{f}\right)^{2}-\left(E\left(\mathbf{p}_{i}\right)-E\left(\mathbf{p}_{f}\right)\right)^{2}$, and we have assumed $t=x_{0}-y_{0}<T / 2$, and $t^{\prime}=$ $y_{0}-z_{0}<T / 2$, and used $\sinh \left(E\left(\mathbf{p}_{i}\right)(t-T / 2)\right) \sim-\cosh \left(E\left(\mathbf{p}_{i}\right)(t-T / 2)\right)+\mathscr{O}\left(e^{E\left(\mathbf{p}_{i}\right)(t-T / 2)}\right)$, and a similar relation for $\sinh \left(E\left(\mathbf{p}_{f}\right)\left(t^{\prime}-T / 2\right)\right)$.

Therefore, the ratio of the three-point and two two-point correlators,

$$
\begin{aligned}
& R_{V}\left(t, t^{\prime} ;\left|\mathbf{p}_{i}\right|,\left|\mathbf{p}_{f}\right|, q^{2}\right) \equiv \frac{\frac{1}{N_{\left|\mathbf{p}_{i}\right|,\left|\mathbf{p}_{f}\right|}^{3 \mathrm{pt}}} \sum_{\text {fixed }\left|\mathbf{p}_{i}\right|,\left|\mathbf{p}_{f}\right|, q^{2}} \frac{C_{P V P}^{3 \mathrm{pt}}\left(t, t^{\prime} ; \mathbf{p}_{i}, \mathbf{p}_{f}\right)}{E\left(\mathbf{p}_{i}\right)+E\left(\mathbf{p}_{f}\right)}}{\left(\frac{1}{N_{\left|\mathbf{p}_{i}\right|}^{2 \mathrm{pt}}} \sum_{\text {fixed }\left|\mathbf{p}_{i}\right|} C_{P P}^{2 \mathrm{pt}}\left(t, \mathbf{p}_{i}\right)\right)\left(\frac{1}{N_{\left|\mathbf{p}_{f}\right|}^{2 \mathrm{pt}}} \sum_{\text {fixed }\left|\mathbf{p}_{f}\right|} C_{P P}^{2 \mathrm{pt}}\left(t^{\prime}, \mathbf{p}_{f}\right)\right)} \\
& =\frac{B}{Y^{2}} F_{V}\left(q^{2}\right)+\cdots,
\end{aligned}
$$

is a good quantity to extract the form factor. Note that the rotationally symmetric correlators are averaged $\left(N_{\left|\mathbf{p}_{i}\right|,\left|\mathbf{p}_{f}\right|}^{3 \mathrm{pt}}\right.$ and $N_{\left|\mathbf{p}_{i}\right|}^{2 \mathrm{pt}}$ denote the numbers of correlators in the summations).

Case 2: $\mathbf{p}_{i} \neq 0, \mathbf{p}_{f}=0\left(\right.$ or $\left.\mathbf{p}_{i}=0, \mathbf{p}_{f} \neq 0\right)$

Let us next consider the case with $\mathbf{p}_{f}=0$, but the initial momentum is kept non-zero. (the opposite case with $\mathbf{p}_{i}=0, \mathbf{p}_{f} \neq 0$, is obtained by simply replacing $\mathbf{p}_{f}=-\mathbf{p}_{i}$ ).

In this case, the contribution from the first term of Eq. (W. I) remains. It is, however, easy to cancel it by taking a subtraction:

$$
\begin{aligned}
\Delta_{t^{\prime}} C_{P V P}^{3 \mathrm{pt}}\left(t, t^{\prime} ; \mathbf{p}_{i}, 0\right) & \equiv C_{P V P}^{3 \mathrm{pt}}\left(t, t^{\prime} ; \mathbf{p}_{i}, 0\right)-C_{P V P}^{3 \mathrm{pt}}\left(t, t_{\mathrm{ref}} ; \mathbf{p}_{i}, 0\right) \\
= & B F_{V}\left(q^{2}\right)\left(\frac{\cosh \left(E\left(\mathbf{p}_{i}\right)(t-T / 2)\right)}{2 E\left(\mathbf{p}_{i}\right) \sinh \left(E\left(\mathbf{p}_{i}\right) T / 2\right)}\right) \\
\times & {\left[-\left(h_{1}^{\prime}\left(\frac{t^{\prime}}{T}\right)-h_{1}^{\prime}\left(\frac{t_{\mathrm{ref}}}{T}\right)\right)+E\left(\mathbf{p}_{i}\right) T\left(h_{1}\left(\frac{t^{\prime}}{T}\right)-h_{1}\left(\frac{t_{\mathrm{ref}}}{T}\right)\right)\right]+\cdots }
\end{aligned}
$$

where

$$
h_{1}(\tau) \equiv \frac{1}{2}\left(\tau-\frac{T}{2}\right)^{2}-\frac{1}{24},
$$

and $h_{1}^{\prime}(\tau)$ is its $\tau$-derivative. The reference time $t_{\text {ref }}$ can be taken arbitrarily.

For having a long-range correlation due to the zero-mode, and a periodic temporal extent, the correlator may have contamination from the $\left\langle\operatorname{vac}\left|V_{0}\right| \pi \pi\right\rangle$ matrix element wrapping around the lattice. In fact, its contribution is not negligible at the leading order of ChPT. But including the next-to-leading order corrections, it is suppressed by a factor $\sim e^{-2 m_{\pi}\left|t^{\prime}-T / 2\right|}$, and, therefore, neglected here. 
Using $C_{P P}^{2 \mathrm{pt}}(t, 0) \propto T h_{1}(t / T)$ and its $t$-derivative defined by $\partial C_{P P}^{2 \mathrm{pt}}(t, 0) \propto h_{1}^{\prime}(t / T)$ in the $\varepsilon$ regime, let us define

$$
R_{V}^{1}\left(t, t^{\prime} ;\left|\mathbf{p}_{i}\right|, 0, q^{2}\right) \equiv \frac{\frac{1}{N_{\left|\mathbf{p}_{i}\right| \text { fixed }\left|\mathbf{p}_{i}\right|, q^{2}}^{3 \mathrm{pt}}}\left(C_{P V P}^{3 \mathrm{pt}}\left(t, t^{\prime} ; \mathbf{p}_{i}, 0\right)-C_{P V P}^{3 \mathrm{pt}}\left(t, t_{\mathrm{ref}} ; \mathbf{p}_{i}, 0\right)\right)}{\frac{1}{N_{\left|\mathbf{p}_{i}\right|}^{2 \mathrm{pt}}} \sum_{\text {fixed }\left|\mathbf{p}_{i}\right|} C_{P P}^{2 \mathrm{pt}}\left(t, \mathbf{p}_{i}\right)\left[-\Delta_{t^{\prime}} \partial C_{P P}^{2 \mathrm{pt}}\left(t^{\prime}, 0\right)+E\left(\mathbf{p}_{i}\right) \Delta_{t^{\prime}} C_{P P}^{2 \mathrm{pt}}\left(t^{\prime}, 0\right)\right]},
$$

where $\Delta_{t^{\prime}} \partial C_{P P}^{2 \mathrm{pt}}\left(t^{\prime}, 0\right) \equiv \partial C_{P P}^{2 \mathrm{pt}}\left(t^{\prime}, 0\right)-\partial C_{P P}^{2 \mathrm{pt}}\left(t_{\mathrm{ref}}, 0\right)$, and $\Delta_{t^{\prime}} C_{P P}^{2 \mathrm{pt}}\left(t^{\prime}, 0\right) \equiv C_{P P}^{2 \mathrm{pt}}\left(t^{\prime}, 0\right)-C_{P P}^{2 \mathrm{pt}}\left(t_{\mathrm{ref}}, 0\right)$, which is again expected to be $B F_{V}\left(q^{2}\right) / Y^{2}$ at large separations of $t$, and $t^{\prime}$. Here, $q^{2}=p_{i}^{2}$.

Case 3: $\mathbf{p}_{i}=\mathbf{p}_{f}=0$

Now it is not difficult to see

$$
R_{V}^{2}\left(t, t^{\prime} ; 0,0,0\right) \equiv \frac{\Delta_{t} \Delta_{t^{\prime}} C_{P V P}^{3 \mathrm{pt}}\left(t, t^{\prime} ; 0,0\right)}{-\Delta_{t} C_{P P}^{2 \mathrm{pt}}(t, 0) \Delta_{t^{\prime}} \partial C_{P P}^{2 \mathrm{pt}}\left(t^{\prime}, 0\right)-\Delta_{t} \partial C_{P P}^{2 \mathrm{pt}}(t, 0) \Delta_{t^{\prime}} C_{P P}^{2 \mathrm{pt}}\left(t^{\prime}, 0\right)},
$$

where we have defined $\Delta_{t} \Delta_{t^{\prime}} C_{P V P}^{3 \mathrm{pt}}\left(t, t^{\prime} ; 0,0\right) \equiv C_{P V P}^{3 \mathrm{pt}}\left(t, t^{\prime} ; 0,0\right)-C_{P V P}^{3 \mathrm{pt}}\left(t, t_{\mathrm{ref}} ; 0,0\right)$ $-C_{P V P}^{3 \mathrm{pt}}\left(t_{\mathrm{ref}}, t^{\prime} ; 0,0\right)+C_{P V P}^{3 \mathrm{pt}}\left(t_{\mathrm{ref}}, t_{\mathrm{ref}} ; 0,0\right)$, is a good quantity to extract $B F_{V}(0) / Y^{2}$. Fortunately, there is no contamination from the $\left\langle\operatorname{vac}\left|V_{0}\right| \pi \pi\right\rangle$ matrix element in this correlator.

Since $R_{V}, R_{V}^{1}$, and $R_{V}^{2}$ share the same overall factor, $B / Y^{2}$, one can eliminate it by taking their ratios. Noting $F_{V}(0)=1$, we find the following two independent extractions

$$
F_{V}\left(t, t^{\prime}, q^{2}\right) \equiv \frac{R_{V}\left(t, t^{\prime} ;\left|\mathbf{p}_{i}\right|,\left|\mathbf{p}_{f}\right|, q^{2}\right)}{R_{V}^{2}\left(t, t^{\prime} ; 0,0,0\right)}, \quad F_{V}^{1}\left(t, t^{\prime}, q^{2}\right) \equiv \frac{R_{V}^{1}\left(t, t^{\prime} ;\left|\mathbf{p}_{i}\right|, 0, q^{2}\right)}{R_{V}^{2}\left(t, t^{\prime} ; 0,0,0\right)},
$$

with $t_{\text {ref }}=T / 4=12$ (on our lattice), are numerically good choices, in that the signals look clean, while the contamination from the excited states is expected to be small.

\section{Preliminary lattice results}

Our numerical simulations are performed with the Iwasaki gauge action at $\beta=2.3$ and $2+1 \mathrm{dy}$ namical flavors of overlap quark action on a $16^{3} \times 48$ lattice. The lattice cutoff $1 / a=1.759(8)(5) \mathrm{GeV}$ $(a \sim 0.112(1) \mathrm{fm}$ ) is determined from the $\Omega$-baryon mass. The physical lattice size is $L \sim 1.8 \mathrm{fm}$.

In this work, we focus on an ensemble with the smallest up-down quark mass, $m a=0.002$. This value roughly corresponds to $3 \mathrm{MeV}$ and the pion mass is $m_{\pi} \sim 99 \mathrm{MeV}$ [目] which is below the physical point. For the strange quark, we choose its mass almost at the physical value, $m_{s} a=$ 0.080. Note in this set up, the pions are in the $\varepsilon$ regime $\left(m_{\pi} L \sim 0.90\right)$.

In the Hybrid Monte Carlo (HMC) updates, the global topological charge of the gauge field is fixed to $Q=0$. Since its effect is encoded in the pion zero-mode, the $Q$ dependence should not appear in the ratios of our correlators.

For the computation of the correlation functions, we use the smeared sources with the form of a single exponential function. To improve the statistical signal, the so-called all-to-all propagator technique is used: the low energy part of the correlator is separately calculated by the 160 

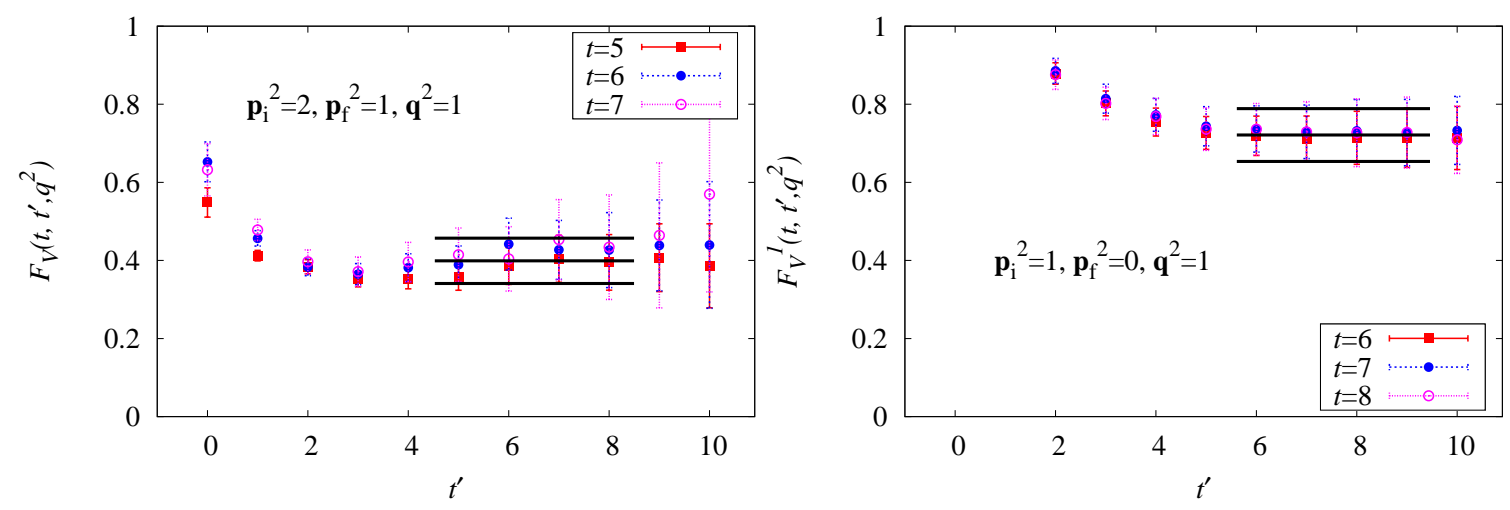

Figure 1: $F_{V}\left(t, t^{\prime}, q^{2}=-0.11\left[\mathrm{GeV}^{2}\right]\right)$ (left panel) and $F_{V}^{1}\left(t, t^{\prime}, q^{2}=-0.40\left[\mathrm{GeV}^{2}\right]\right)$ (right).

eigenmodes of the Dirac operator and averaged over different source points, and the higher-mode contribution is estimated stochastically by the noise method with the dilution technique.

Details of the numerical simulation will be reported elsewhere.

Figure凹shows our lattice data of $F_{V}\left(t, t^{\prime}, q^{2}\right)$ at $\left(\mathbf{p}_{i}^{2}, \mathbf{p}_{f}^{2}, \mathbf{q}^{2}\right)=(2,1,1)$ (left panel) and $F_{V}^{1}\left(t, t^{\prime}, q^{2}\right)$ at $\left(\mathbf{p}_{i}^{2}, \mathbf{p}_{f}^{2}, \mathbf{q}^{2}\right)=(1,0,1)$ (right panel). Here, the momenta are denoted in units of $2 \pi / L$. To estimate $E(\mathbf{p})$, we have used the dispersion relation $E(\mathbf{p})=\sqrt{\mathbf{p}^{2}+m_{\pi}^{2}}$. Each set of data shows a plateau around $t, t^{\prime}=5-9$ from which we extract $F_{V}\left(q^{2}\right)$ by a constant fit.

The $q^{2}$ dependence of $F_{V}\left(q^{2}\right)$ is presented in Fig. 미. For comparison, we have also plotted data in the $p$ regime (at $m=0.015$ ) [12]. Our new data in the $\varepsilon$ regime shows a steeper slope near the origin, which results in a large value of the pion charge radius,

$$
\left.\left\langle r^{2}\right\rangle_{V} \equiv 6 \frac{\partial F_{V}\left(q^{2}\right)}{\partial q^{2}}=0.53(4) \mathrm{fm}^{2} \text { [preliminary }\right]
$$

This value is obtained by fitting the data to a function $F_{V}\left(q^{2}\right)=\frac{1}{1-q^{2} / m_{\rho}^{2}}+a_{1} q^{2}+a_{2}\left(q^{2}\right)^{2}$ [ए]], (solid curve in Fig. (2) with an input of the rho-meson mass $m_{\rho}=770 \mathrm{MeV}$ (a fit without rhomeson contribution (dashed curve) is also tried). This result is larger than the experimental value $\left(0.452(11) \mathrm{fm}^{2}\right)$ and confirms the existence of the strong (logarithmic) curvature of the pion charge radius near the chiral limit, as shown in Fig. [1].

In this work, we have demonstrated how to cancel finite volume effects in the $\varepsilon$ regime. Inserting momenta to the operators, and taking appropriate ratios of them, we can eliminate the contribution from the pion zero-mode. This cancellation occurs only at the leading order, and there should be non-trivial corrections from NLO terms. But it is suppressed by a factor $\sim 1 / 4 \pi F^{2} \sqrt{V}$ ( $\sim 7 \%$ on our lattice). Our tree-level analysis of the vector pion form factor on the lattice in the $\varepsilon$ regime confirms the above observation and our preliminary result for the pion charge radius is consistent with the experiment, showing the existence of a diverging logarithmic curve.

In this work, we have focused on the calculation of the vector (or electro-magnetic) form factor. But in principle, this method can be applied for any other form factors. 
We thank P. H. Damgaard for useful discussions. Numerical simulations are performed on the IBM System Blue Gene Solution at High Energy Accelerator Research Organization (KEK) under a support of its Large Scale Simulation Program (No. 09/10-09, 11-05). This work is supported in part by the Grant-in-Aid of the Japanese Ministry of Education (No. 21674002, 22740183), the Grant-in-Aid for Scientific Research on Innovative Areas (No. 2004: 20105001, 20105002, 20105003, 20105005, 23105710), and SPIRE (Strategic Program for Innovative Research).

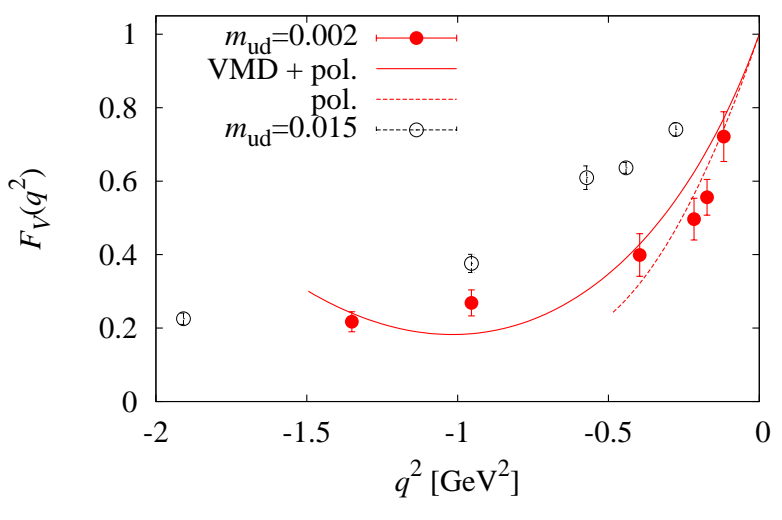

Figure 2: The $q^{2}$ dependence of $F_{V}\left(q^{2}\right)$.

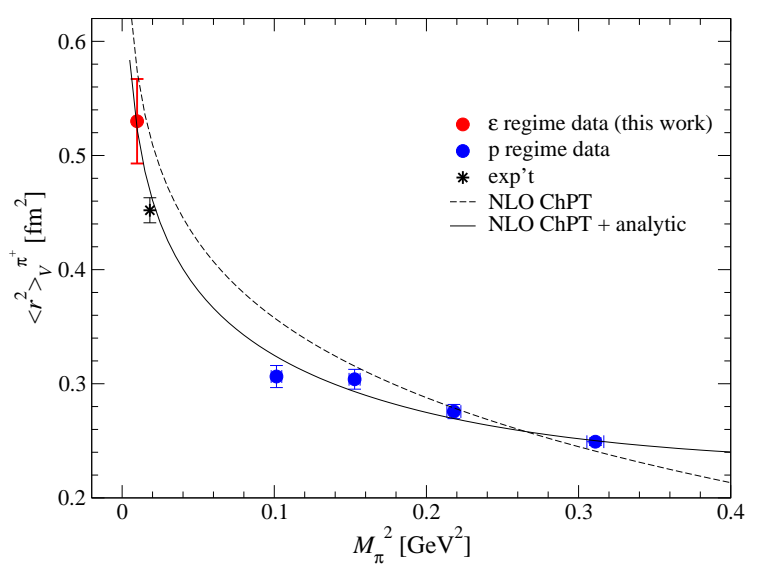

Figure 3: The $m_{\pi}^{2}$ dependence of the pion charge radius.

\section{References}

[1] H. Neuberger, Phys. Lett. B 417, 141 (1998), Phys. Lett. B 427, 353 (1998).

[2] H. Fukaya et al. [JLQCD Collaboration], Phys. Rev. Lett. 98, 172001 (2007).

[3] H. Fukaya et al. [JLQCD collaboration], Phys. Rev. D 77, 074503 (2008).

[4] H. Fukaya et al. [JLQCD collaboration], Phys. Rev. Lett. 104, 122002 (2010) [Erratum-ibid. 105, 159901 (2010)]; JLQCD and TWQCD collaborations, Phys. Rev. D 83, 074501 (2011).

[5] H. Fukaya et al. [JLQCD Collaboration], arXiv:1111.0417 [hep-lat].

[6] J. Gasser and H. Leutwyler, Phys. Lett. B 184, 83 (1987).

[7] P. H. Damgaard and H. Fukaya, JHEP 0901, 052 (2009). [arXiv:0812.2797 [hep-lat]].

[8] S. Aoki and H. Fukaya, Phys. Rev. D 84, 014501 (2011) [arXiv:1105.1606 [hep-lat]].

[9] L. Giusti, P. Hernandez, M. Laine, P. Weisz and H. Wittig, JHEP 0411, 016 (2004) [hep-lat/0407007].

[10] S. Aoki et al. [JLQCD and TWQCD Collaboration], Phys. Rev. D 80, 034508 (2009).

[11] F. Bernardoni, P. H. Damgaard, H. Fukaya and P. Hernandez, JHEP 0810, 008 (2008).

[12] T. Kaneko et al.[JLQCD Collaboration], PoS LATTICE2010, 146 (2010). 